\title{
Neutrino-Electron Elastic Scattering and Stellar Energy Loss Through Electron-Positron Pair Annihilation in the Magnetic Model.
}

C. AYDin

Karadeniz Technical University, Department of Physics - 61080 Trabzon, Turkey

(Nuovo Cimento A, 105, 843 (1992))

PACS 14.60.Gh - Neutrinos and other neutral leptons.

PACS 99.10 - Errata.

On p. 847 , eq. (18), eq. (19), eq. (20) and eq. (21) should be

$$
\begin{aligned}
& \sigma_{\mathrm{tat}}=\frac{K_{\mathrm{y}}^{2}}{2 \pi s^{2}\left(s-4 m^{2}\right)}\left\{\frac{e^{2}}{6}\left[2 t^{3}+3 t^{2}\left(s-2 m^{2}\right)+6\left(m^{4}-s m^{2}\right) t\right]-\right. \\
& \left.\quad-e m K_{\mathrm{e}} s^{2} t-\frac{s}{12} K_{\mathrm{e}}^{2}\left[3 s^{2} t+6 s t^{2}+4 t^{3}-12 m^{2} t^{2}+12 m^{2} t\right]\right\}\left.\right|_{t_{\min }} ^{t_{\max }} .
\end{aligned}
$$

$$
\begin{aligned}
\sigma=\frac{K_{v}^{2}}{12 \pi E_{+} E_{-} v}\left\{2 e^{2} m^{2}+2 m^{4}\right. & K_{\mathrm{e}}^{2}+6 e m^{3} K_{\mathrm{e}}+ \\
& \left.+\left(e^{2}+6 e m K_{\mathrm{e}}+6 m^{2} K_{\mathrm{e}}^{2}\right) p_{1} p_{2}+K_{\mathrm{e}}^{2}\left(p_{1} p_{2}\right)^{2}\right\} .
\end{aligned}
$$

$$
\begin{gathered}
Q=\frac{4}{(2 \pi)^{6}} \int \frac{\mathrm{d}^{3} \boldsymbol{p}_{-}}{\exp \left[\left(E_{-}-\mu\right) / k T\right]+1} \frac{\mathrm{d}^{3} \boldsymbol{p}_{+}}{\exp \left[\left(E_{+}+\mu\right) / k T\right]+1}\left(E_{+}^{+} E_{-}\right) v \sigma . \\
Q_{\mathrm{I}}^{\mathrm{mag}}=\frac{K_{\nu}^{2} m^{7}}{4 \pi^{4}}\left(e^{2}+3 m^{2} K_{\mathrm{e}}^{2}+4 e m K_{\mathrm{e}}\right)\left(\frac{k T}{m}\right)^{3} \exp \left[\frac{-2 m}{k T}\right] .
\end{gathered}
$$

On. p. 848 , eq. (22) and eq. (26) should be

$$
\begin{gathered}
Q_{\mathrm{II}}^{\mathrm{mag}}=\frac{K_{v}^{2} \sqrt{2 \pi} m^{4}}{4 \pi^{3}}\left(e^{2}+3 m^{2} K_{\mathrm{e}}^{2}+4 e m K_{\mathrm{e}}\right)\left(\frac{k T}{m}\right)^{3 / 2}\left(\frac{N_{\rho}}{\mu_{\mathrm{e}}}\right) \exp \left[\frac{-m-\mu}{k T}\right], \\
\frac{Q_{\mathrm{I}, \mathrm{II}}^{\mathrm{mag}}}{Q_{\mathrm{I}, \mathrm{II}}^{\text {stand }}}=\frac{K_{\mathrm{e}}^{2}\left(e^{2}+3 m^{2} K_{\mathrm{e}}^{2}+4 e m K_{\mathrm{e}}\right)}{4 G^{2} C_{V}^{2} m^{2}} .
\end{gathered}
$$

On p. 848 , in conclusion

$$
K_{\nu}^{2}\left(e^{2}+K_{\mathrm{e}}^{2}+3 m^{2} K_{\mathrm{e}}^{2}+4 e m K_{\mathrm{e}}\right) / 4 G^{2} C_{V}^{2} m^{2}
$$

should be

$$
K_{v}^{2}\left(e^{2}+3 m^{2} K_{\mathrm{e}}^{2}+4 e m K_{\mathrm{e}}\right) / 4 G^{2} C_{V}^{2} m^{2} .
$$

